\title{
BOUNDS ON THE NUMBER OF EULERIAN ORIENTATIONS
}

\author{
A. SCHRIJVER \\ Dedicated to Paul Erdös on his seventieth birthday
}

Received 3 February 1983

We show that each loopless $2 k$-regular undirected graph on $n$ vertices has at least $\left(2^{-k}\left(\begin{array}{c}2 k \\ k\end{array}\right)\right)^{n}$ and at most $\sqrt{\left(\begin{array}{c}2 k \\ k\end{array}\right)^{n}}$ eulerian orientations, and that, for each fixed $k$, these ground numbers are best possible.

Let for any undirected graph $G=(V, E), \varepsilon(G)$ denote the number of eulerian orientations of $G$. Here an eulerian orientation is an orientation of the edges so that in each vertex the indegree is equal to the outdegree. We are interested in upper and lower bounds for $\varepsilon(G)$ in terms of the degree sequence of $G$. We show that if $G$ is a loopless $2 k$-regular undirected graph on $n$ vertices, then

$$
\left(2^{-k}\left(\begin{array}{c}
2 k \\
k
\end{array}\right)\right)^{n} \leqq \varepsilon(G) \leqq\left(\sqrt{\left(\begin{array}{c}
2 k \\
k
\end{array}\right)}\right)^{n},
$$

and moreover that for each fixed $k$ the ground numbers in (1), as functions of $k$ only, cannot be improved. That is,

(2) $\quad \inf _{G} \varepsilon(G)^{1 /|V(G)|}=2^{-k}\left(\begin{array}{c}2 k \\ k\end{array}\right) \quad$ and $\quad \sup _{G} \varepsilon(G)^{1 /|V(G)|}=\sqrt{\left(\begin{array}{c}2 k \\ k\end{array}\right)}$,

where the infimum and supremum range over all loopless $2 k$-regular graphs $G$, and where $V(G)$ denotes the vertex set of $G$. It is easy to see that in (2) inf and sup may be replaced by lim inf and lim sup.

There is a direct relation between $\varepsilon(G)$ and the matrix permanent function. If $G=(V, E)$ is an undirected graph where each vertex $v$ of $G$ has degree $\operatorname{deg}(v)$ even, and if $B$ is the incidence matrix of $G$ (with $|V|$ rows and $|E|$ columns), let the matrix $A$ arise from $B$ by repeating, for each vertex $v$, the row corresponding to $v 1 / 2 \operatorname{deg}(v)$ times. This matrix $A$ then is a square matrix of order $|E|$. Now it is easy 
to see that

$$
\varepsilon(G)=\frac{\operatorname{per} A}{\prod_{v \in V}\left(\frac{1}{2} \operatorname{deg}(v)\right) !} .
$$

The upper bound in (1) follows now easily from Brègman's result [1] (Minc's conjecture [7], cf. [8]), saying that if $A$ is a square $(0,1)$-matrix of order $m$, with row sums $r_{1}, \ldots, r_{m}$, then

$$
\operatorname{per} A \leqq \prod_{i=1}^{m} r_{i} ! 1 / r_{i}
$$

Substitution of (4) in (3) gives

$$
\varepsilon(G) \equiv \prod_{v \in V}\left(\begin{array}{c}
\operatorname{deg}(v) \\
\frac{1}{2} \operatorname{deg}(v)
\end{array}\right)^{\frac{1}{2}},
$$

and the upper bound in (1) follows. The graph with two vertices connected by $2 k$ parallel edges shows that we cannot have a lower ground number in this upper bound.

Concerning lower bounds, Falikman [4] and Egorychev [2] proved Van der Waerden's conjecture [11], which can be formulated as: if $A$ is a nonnegative square matrix of order $m$, with all row and column sums equal to $r$, then per $A \geqq\left(\frac{r}{m}\right)^{m} m !$. Substitution in (3) gives

$$
\varepsilon(G) \geqq\left(\frac{2}{n}\right)^{n k} \cdot \frac{(n k) !}{k !^{n}}
$$

if $G$ is a $2 k$-regular undirected graph on $n$ vertices. Asymptotically this implies

In [9] it is conjectured that

$$
\varepsilon(G) \geqq\left(\frac{1}{k !}\left(\frac{2 k}{e}\right)^{k}\right)^{n}
$$

$$
\operatorname{per} A \geqq\left(\frac{(r-1)^{r-1}}{r^{r-2}}\right)^{m}
$$

if $A$ is a square nonnegative integral matrix of order $m$ with all row and column sums equal to $r$. (This bound was proved for $r=3$ by Voorhoeve [10], thereby showing a conjecture of Erdós and Rényi [3], and was shown to have the best possible gromn. number as a function of $r$ in [12] and [9].) Conjecture (8) would imply that

$$
\varepsilon(G) \geqq\left(\frac{1}{k !} \frac{(2 k-1)^{2 k-1}}{(2 k)^{2 k-2}}\right)^{n},
$$

if $G$ is $2 k$-regular. The lower bound for $\varepsilon(G)$ given in (1) is higher than that in (9). This is not surprising, as generally the permanent function seems to approach its minimum if the matrix tends to have a random structure, whereas the matrix $\boldsymbol{A}$ derived from the incidence matrix of $G$ has several equal rows.

Actually we show a slightly more general result on the lower bound for $\varepsilon(G)$ for not-necessarily regular graphs. It will turn out that the lower bound in (1) also holds for not-loopless $2 k$-regular graphs. Here, by convention, a loop is counted for 
2 in the degree, and it can be oriented in two directions. (Clearly, we may not skip "loopless" at the upper bound, as the graph $G$ consisting of one vertex attached with $k$ loops has $\varepsilon(G)=2^{k}$.)

Define, for natural numbers $k_{1}, \ldots, k_{n}$,

$$
E\left(2 k_{1}, \ldots, 2 k_{n}\right):=\min _{G} \varepsilon(G)
$$

where the minimum ranges over all undirected graphs $G$ with degree sequence $2 k_{1}, \ldots$ $\ldots, 2 k_{n}$.

Theorem 1. $\frac{1}{2^{m}}\left(\begin{array}{c}2 k_{1} \\ k_{1}\end{array}\right) \cdot \ldots \cdot\left(\begin{array}{c}2 k_{n} \\ k_{n}\end{array}\right) \leqq E\left(2 k_{1}, \ldots, 2 k_{n}\right) \leqq \frac{2^{m}}{\left(\begin{array}{c}2 m \\ m\end{array}\right)}\left(\begin{array}{c}2 k_{1} \\ k_{1}\end{array}\right) \cdot \ldots \cdot\left(\begin{array}{c}2 k_{n} \\ k_{n}\end{array}\right)$, for any natural numbers $k_{1}, \ldots, k_{n}$, where $m:=k_{1}+\ldots+k_{n}$ (the number of edges).

Proof. Without loss of generality we may assume that no $k_{i}$ is equal to 0 .

I. We prove the lower bound by induction on $\sum_{i=1}^{n}\left(k_{i}-1\right)$, the bound being trivial if $k_{1}=\ldots=k_{n}=1$. Assume $k_{n} \geqq 2$, and let $G$ be a graph with degree sequence $2 k_{1}, \ldots, 2 k_{n}$, and with $E\left(2 k_{1}, \ldots, 2 k_{n}\right)$ eulerian orientations. Let $v$ be a vertex with degree $2 k_{n}$, and let $e_{1}, \ldots, e_{2 k_{n}}$ be the edges incident with $v$. Let for all $1 \leqq i<j \leqq 2 k_{n}$, $\varepsilon_{i j}$ denote the number of those eulerian orientations of $G$ in which $e_{i}$ and $e_{j}$ are oriented in series (i.e., either $e_{i}$ enters $v$ and $e_{j}$ leaves $v$, or conversely). So, by replacing the edges $e_{i}=\left\{v, v_{1}\right\}$ and $e_{j}=\left\{v, v_{2}\right\}$ by the new edges $e_{i}^{\prime}:=\left\{v^{\prime}, v_{1}\right\}$ and $e_{j}^{\prime}:=\left\{v^{\prime}, v_{2}\right\}$, where $v^{\prime}$ is a new vertex, we obtain a graph $G^{\prime}$ with degree sequence $2 k_{1}, \ldots, 2 k_{n-1}$, $2 k_{n}-2,2$, such that $\varepsilon_{i j}$ is equal to $\varepsilon\left(G^{\prime}\right)$. Hence, by induction,

$$
\varepsilon_{i j} \geqq E\left(2 k_{1}, \ldots, 2 k_{n-1}, 2 k_{n}-2,2\right) \geqq \frac{1}{2^{m}}\left(\begin{array}{c}
2 k_{1} \\
k_{1}
\end{array}\right) \cdot \ldots \cdot\left(\begin{array}{c}
2 k_{n-1} \\
k_{n-1}
\end{array}\right)\left(\begin{array}{c}
2 k_{n}-2 \\
k_{n}-1
\end{array}\right) \cdot 2
$$

for all $1 \leqq i<j \leqq 2 k_{n}$. Moreover,

$$
\sum_{i<j} \varepsilon_{i j}=k_{n}^{2} \cdot \varepsilon(G)=k_{n}^{2} \cdot E\left(2 k_{1}, \ldots, 2 k_{n}\right)
$$

as for each eulerian orientation of $G$ there are $k_{n}^{2}$ choices $i, j(i<j)$ for which it is counted in $\varepsilon_{i j}$.

Combining (11) and (12) we obtain the lower bound on $E\left(2 k_{1}, \ldots, 2 k_{n}\right)$.

II. In order to prove the upper bound, let $k_{1}, \ldots, k_{n}$ be natural numbers, let $m=k_{1}+\ldots+k_{n}$, and let $G=(V, E)$ be a 1-regular undirected graph with $|V|$ $=2 m$ and $|E|=m$. Let $\mathscr{P}$ be the collection of partitions $\left(V_{1}, \ldots, V_{n}\right)$ of $V$ such that $\left|V_{1}\right|=2 k_{1}, \ldots,\left|V_{n}\right|=2 k_{n}$. Define for each partition $\Pi=\left(V_{1}, \ldots, V_{n}\right)$ in $\mathscr{P}$ the graph $G_{\Pi}$ which arises from $G$ by, successively, contracting $V_{1}, \ldots, V_{n}$, thus obtaining a graph with $n$ vertices and degree sequence $2 k_{1}, \ldots, 2 k_{n}$ (if an edge of $G$ is contained in one of the classes $V_{i}$, it yields a loop in $\left.G_{n}\right)$.

Now we evaluate the sum $\sum_{n \in \mathscr{P}} \varepsilon\left(G_{\not I}\right)$ in two ways. First

$$
\sum_{\Pi \in \mathscr{P}} \varepsilon\left(G_{\Pi}\right) \geqq|\mathscr{P}| \cdot E\left(2 k_{1}, \ldots, 2 k_{n}\right)=\left(\begin{array}{c}
2 m \\
2 k_{1}, \ldots, 2 k_{n}
\end{array}\right) \cdot E\left(2 k_{1}, \ldots, 2 k_{n}\right) .
$$


Second, since each orientation of the edges of $G$ induces a unique orientation of the edges of $G_{I I}$,

$$
\begin{gathered}
\sum_{n \in,} \delta\left(G_{n}\right)=\sum_{\text {norientation of } G} \text { (number of } \Pi \text { in } \not \text { such that }, \text { is an } \\
\text { eulerian orientation of } \left.G_{\Pi}\right)=2^{m}\left(\begin{array}{c}
m \\
k_{1}, \ldots, k_{n}
\end{array}\right)^{2} .
\end{gathered}
$$

This follows from the fact the $G$ has $2^{m}$ orientations, while for any fixed orientation cof the edges of $G$ there are $\left(k_{1}, \ldots, k_{n}\right)^{2}$ partitions $\Pi$ in $\not y$ such that $\%$ is an eulerian orientation of $G_{I T}$. Indeed, we must choose in the first class $k_{1}$ heads and $k_{1}$ tails, in the second class $k_{2}$ heads and $k_{2}$ tails, and so on.

Combination of (13) and (14) gives the upper bound in Theorem 1.

Corollary 1a. Each $2 k$-regular undirected graph on $n$ vertices has at least $\left(2^{-k}\left(\begin{array}{c}2 k \\ k\end{array}\right)\right)^{n}$ eulerian orientations. For fixed $k$ the ground number is best possible.

Proof. Taking $k_{1}=\ldots=k_{n}=k$, and taking the $n$-th root of both bounds in Theorem 1 , gives with Stirling's asymptotical formula the required result.

In fact, also if we restrict ourselves to loopless graphs, the ground number in this corollary cannot be increased.

Theorem 2. For each natural number $k$, let $f(2 k)$ be the highest possible number such that each loopless $2 k$-regular undirected graph on $n$ vertices has at least $f(2 k)^{n}$ eulerian orientations. Then $f(2 k)=2^{-k}\left(\begin{array}{c}2 k \\ k\end{array}\right)$.

Proof. The inequality $f(2 k) \geqq 2^{-k}\left(\begin{array}{c}2 k \\ k\end{array}\right)$ directly follows from Corollary la. To show the converse inequality, we first show that if $G=(V, E)$ is an undirected graph with all degrees even and $e^{\prime}=\{v, w\}$ and $e^{\prime \prime}=\{v, w\}$ are parallel edges, and if we denote

$$
\begin{aligned}
\varepsilon^{\prime}(G):= & \text { the number of eulerian orientations of } G \text { in which } e^{\prime} \text { and } e^{\prime \prime} \text { have the } \\
& \text { same orientation, } \\
\varepsilon^{\prime \prime}(G):= & \text { the number of eulerian orientations of } G \text { in which } e^{\prime} \text { and } e^{\prime \prime} \text { have } \\
& \text { opposite orientations, }
\end{aligned}
$$

(so $\left.\varepsilon(G)=\varepsilon^{\prime}(G)+\varepsilon^{\prime \prime}(G)\right)$, then $\varepsilon^{\prime}(G) \equiv \varepsilon^{\prime \prime}(G)$. This could be proved using the "Alexandroff-Fenchel permanent inequality" (used by Falikman and Egorychev to prove the Van der Waerden conjecture-cf. [5], [6]), but here we give a direct proof. The inequality is proved by induction on $|E|$. Choose a vertex $u \neq v, w$. (If no such vertex exists the inequality is easy.) Let $c_{1}, \ldots, c_{2 k}$ be the edges incident with $u$, and define, for $1 \leqq i<j \leqq 2 k$,

$$
\begin{aligned}
\varepsilon_{i j}^{\prime}(G)= & \text { the number of eulerian orientations of } G \text { in which } e^{\prime} \text { and } e^{\prime \prime} \text { have the } \\
& \text { same orientation, and in which } e_{i} \text { and } e_{j} \text { are oriented in series; } \\
\varepsilon_{i j}^{\prime \prime}(G)= & \text { the number of eulerian orientations of } G \text { in which } e^{\prime} \text { and } e^{\prime \prime} \text { have } \\
& \text { opposite orientations, and in which } e_{i} \text { and } e_{j} \text { are oriented in series. }
\end{aligned}
$$


Then, like in the proof of Theorem 1,

$$
\begin{aligned}
& \varepsilon^{\prime}(G)=k^{-2} \cdot \sum_{1 \leqq i=j \leqq 2 k} \varepsilon_{i j}^{\prime}(G), \\
& \varepsilon^{\prime \prime}(G)=k^{-2} \cdot \sum_{1 \equiv i<j-52 k} \varepsilon_{i j}^{\prime \prime}(G) .
\end{aligned}
$$

Now for each $i<j, \quad \varepsilon_{i j}^{\prime}(G) \equiv \varepsilon_{i j}^{\prime \prime}(G)$, since the edges $e_{i}=\{s, u\}$ and $e_{j}=\{t, u\}$ can be replaced by one new edge $\{s, t\}$, and then. by induction, for the new graph $G^{\prime}$ we know:

$$
\varepsilon_{i j}^{\prime}(G)=\varepsilon^{\prime}\left(G^{\prime}\right) \equiv \varepsilon^{\prime \prime}\left(G^{\prime}\right)=\varepsilon_{i j}^{\prime \prime}(G) .
$$

Combination of (17) and (18) gives $\varepsilon^{\prime}(G) \leqq \varepsilon^{\prime \prime}(G)$.

In order to show $f(2 k) \leqq 2^{-k}\left(\begin{array}{c}2 k \\ k\end{array}\right)$, we have to show, for any $\delta>0$, the existence of a loopless $2 k$-regular graph $G=(V, E)$ such that

$$
\varepsilon(G) \leqq\left(2^{-k}\left(\begin{array}{c}
2 k \\
k
\end{array}\right)+\delta\right)^{n}
$$

where $n=|v|$.

By Corollary la we know that there exists a $2 k$-regular graph $G=(V, E)$ satisfying (19). Choose such $G$ with as few loops as possible. Suppose $v$ and $w$ are distinct vertices of $G$ having loops. Replace one pair of loops $l^{\prime}=\{v, v\}, l^{\prime \prime}=\{w, w\}$ by two new parallel edges $e^{\prime}=\{v, w\}$ and $e^{\prime \prime}=\{v, w\}$, making up the graph $G^{\prime}$. Let $\varepsilon^{\prime}\left(G^{\prime}\right)$ and $\varepsilon^{\prime \prime}\left(G^{\prime}\right)$ be as defined in (15). Then

$$
\varepsilon(G)=2 \varepsilon^{\prime \prime}\left(G^{\prime}\right) \geqq \varepsilon^{\prime}\left(G^{\prime}\right)+\varepsilon^{\prime \prime}\left(G^{\prime}\right)=\varepsilon\left(G^{\prime}\right) .
$$

Hence also $G^{\prime}$ satisfies (19), contradicting our choice of $G$.

If there is only one vertex $v$ of $G$ with, say $p$ loops, let $G^{\prime}$ arise from $G$ by duplicating $G$ (where $v^{\prime}$ denotes the duplicate of vertex $v$ ), and replacing the $p$ loops attached to $v$ and the $p$ loops attached to $v^{\prime}$ by $2 p$ parallel edges $\left\{v, v^{\prime}\right\}$. Then

$$
\varepsilon\left(G^{\prime}\right)=\left(\begin{array}{c}
2 t \\
t
\end{array}\right) 2^{-2 t} \varepsilon(G)^{2} \equiv \varepsilon(G)^{2} .
$$

As (19) holds for $G$, we know that (19) holds for $G^{\prime}$, with $n$ replaced by $2 n$. As $G^{\prime}$ is loopless, this proves the theorem.

Finally, two conjectures for simple graphs (i.e., without loops or parallel edges). First we conjecture that the lower bound in (1) has best possible ground number also if we restrict ourselves to simple graphs. Second, that the upper bound in (1) can be improved for simple graphs to: if $G$ is a simple undirected graph with degree sequence $2 k_{1}, \ldots, 2 k_{n}$, then

$$
\text { (Conjecture) } \quad \varepsilon(G) \leqq \prod_{i=1}^{n} \varepsilon\left(K_{2 k_{i}+1}\right)^{1 /\left(2 k_{i}+1\right)} .
$$

( $K_{t}$ denotes the complete undirected graph on $t$ vertices.) A problem we met in constructing a proof similar to that of Brègman's upper bound (cf. [8]) is that we could not find a suitable formula for $\varepsilon\left(K_{t}\right)$. 


\section{References}

[1] L. M. Brìgman, Some properties of nonnegative matrices and their permanents, Soviet Math. Dokl. 14 (1973) 945-949 (English translation of: Dokl. Akad. Nauk SSSR 211 (1973) 27-30).

[2] G. P. Egorychev, Solution of Van der Waerden's permanent conjecture, Advances in Math. 42 (1981) 299-305

[3] P. ERdös and A. RÉNYI, On random matrices II, Studia Sci. Math. Hungar. 3 (1968) $459-464$.

[4] D. I. Falikman, A proof of the Van der Waerden conjecture on the permanent of a doubly stochastic matrix, Mat. Zametki 29 (1981) 931-938 (Russian).

[5] D. E. KNuth, A permanent inequality, Amer. Math. Monthly 88 (1981) 731-740.

[6] J. H. VAN LINT, Notes on Egoritsjev's proof of the Van der Waerden conjecture, Linear Algebra and Appl. 39 (1981) $1-8$.

[7] H. Minc, Upper bounds for permanents of (0,1)-matrices, Bull. Amer. Math. Soc. 69 (1963) 789-791.

[8] A. SchriJver, A short proof of Minc's conjecture, J. Combinatorial Theory (A) 25 (1978) 80-83.

[9] A. SchriJver and W. G. Valiant, On lower bounds for permanents, Indag. Math. 42 (1980) $425-427$.

[10] M. VooRHoeve, A lower bound for the permanents of certain (0,1)-matrices, Indag. Math. 41 (1979) 83-86.

[11] B. L. VAN DER Warrden, Aufgabe 45, Jahresber. Deutsch. Math.-Verein. 25 (1926) 117.

[12] H. S. WILF, On the permanent of a doubly stochastic matrix, Canad. J. Math. 18 (1966) $758-761$.

A. Schrijver

Departnent of Econometrics,

Tilburg Uniciersity,

P.O. Box. 90153 ,

5000 LE Tilburg,

The Netherlands

and

Mathematical Centre,

Kruislaan 413, 1098 SJ Amsterdam,

The Netherlands. 